\title{
Video Article \\ Combined Optogenetic and Freeze-fracture Replica Immunolabeling to Examine Input-specific Arrangement of Glutamate Receptors in the Mouse Amygdala
}

\author{
Sabine Schönherr ${ }^{1}$, Anna Seewald ${ }^{1}$, Yu Kasugai ${ }^{1}$, Daniel Bosch ${ }^{2}$, Ingrid Ehrlich ${ }^{2}$, Francesco Ferraguti \\ ${ }^{1}$ Department of Pharmacology, Medical University of Innsbruck \\ ${ }^{2}$ Hertie Institute for Clinical Brain Research and Centre for Integrative Neuroscience, University of Tübingen \\ Correspondence to: Francesco Ferraguti at francesco.ferraguti@i-med.ac.at
}

URL: https://www.jove.com/video/53853

DOI: doi:10.3791/53853

Keywords: Neuroscience, Issue 110, Neuroscience, cell biology, electron microscopy, amygdala, synapse, glutamate receptors

Date Published: 4/15/2016

Citation: Schönherr, S., Seewald, A., Kasugai, Y., Bosch, D., Ehrlich, I., Ferraguti, F. Combined Optogenetic and Freeze-fracture Replica Immunolabeling to Examine Input-specific Arrangement of Glutamate Receptors in the Mouse Amygdala. J. Vis. Exp. (110), e53853, doi:10.3791/53853 (2016)

\section{Abstract}

Freeze-fracture electron microscopy has been a major technique in ultrastructural research for over 40 years. However, the lack of effective means to study the molecular composition of membranes produced a significant decline in its use. Recently, there has been a major revival in freeze-fracture electron microscopy thanks to the development of effective ways to reveal integral membrane proteins by immunogold labeling. One of these methods is known as detergent-solubilized Freeze-fracture Replica Immunolabeling (FRIL).

The combination of the FRIL technique with optogenetics allows a correlated analysis of the structural and functional properties of central synapses. Using this approach it is possible to identify and characterize both pre- and postsynaptic neurons by their respective expression of a tagged channelrhodopsin and specific molecular markers. The distinctive appearance of the postsynaptic membrane specialization of glutamatergic synapses further allows, upon labeling of ionotropic glutamate receptors, to quantify and analyze the intrasynaptic distribution of these receptors. Here, we give a step-by-step description of the procedures required to prepare paired replicas and how to immunolabel them. We will also discuss the caveats and limitations of the FRIL technique, in particular those associated with potential sampling biases. The high reproducibility and versatility of the FRIL technique, when combined with optogenetics, offers a very powerful approach for the characterization of different aspects of synaptic transmission at identified neuronal microcircuits in the brain.

Here, we provide an example how this approach was used to gain insights into structure-function relationships of excitatory synapses at neurons of the intercalated cell masses of the mouse amygdala. In particular, we have investigated the expression of ionotropic glutamate receptors at identified inputs originated from the thalamic posterior intralaminar and medial geniculate nuclei. These synapses were shown to relay sensory information relevant for fear learning and to undergo plastic changes upon fear conditioning.

\section{Video Link}

The video component of this article can be found at https://www.jove.com/video/53853/

\section{Introduction}

The definition of the functional architecture of biomembranes at nanometer scale has been challenged in recent years by the development of a number of immunolabeling techniques suitable for transmission electron microscopy. However, these techniques, e.g., pre- and postembedding immunogold, have a number of important limitations, which include poor detection of antigens and/or limited quantitative assessment of membrane-bound proteins. These limitations become particularly critical in the investigation of the fine structure of the nervous system, which is characterized by a high degree of cell diversity and synapse heterogeneity. This heterogeneity results from both structural and functional diversity dictated by the pre- and postsynaptic elements and by the differential expression, enrichment, or interaction of signalling proteins, such as receptors, transporters, and effector molecules.

A new approach for direct immunolabeling of integral or cross-linked membrane proteins in detergent-solubilized freeze-fracture replicas (FRIL) was originally introduced by Fujimoto two decades ago ${ }^{1}$. This original method had, however, several limitations, i.e., severe fragmentation of replicas, which hampered meaningful correlations of labeled molecules with individually mapped cells in complex tissues such as the brain. Approximately 10 years ago, Shigemoto and Fukazawa progressively improved the technique ${ }^{2}$. This was paralleled by efforts from another group of scientists at the Boulder laboratories of the Colorado State University, who also significantly improved the technique, in particular for the study of gap junctions ${ }^{3}$. 
The improvement in freeze-fracturing protocols and machines, as well as the introduction of quick freezing (under high pressure), now allows investigators to produce unbroken replicas of specimens of relatively large size and high quality images of most cellular components without the limitations and artifacts produced by strong chemical fixations.

The FRIL technique offers the great advantage of a highly quantitative in situ identification of one or more proteins (simultaneously) in histologically mapped and cytologically identified cells within complex tissues such as the brain, with the additional advantage of a planar view of pre- and postsynaptic elements in a single replica. Therefore, the FRIL technique, despite its many technical hurdles, holds the promise for a number of very significant scientific breakthroughs, particularly for the correlation of structural and functional properties of individual synapses. During the last several decades, a great deal of information has been obtained on the structure, molecular make up, and physiological function of synapses; yet synapses are morphologically and molecularly highly diverse depending on the pre and postsynaptic parent neurons ${ }^{4}$. Only for a handful of synapse types were structure-function studies accomplished so far ${ }^{5-7}$. This was mostly due to technical constrains that prevented a precise identification of the nature of the pre- and postsynaptic elements.

Ultrastructural analysis has provided critical insights into the variability of postsynaptic membrane specializations across distinct synaptic contacts both in terms of synaptic size and content in neurotransmitter receptors ${ }^{6}$, which has a large impact on the strength and plasticity of synaptic transmission. Furthermore, a large body of research indicates that the number of ionotropic glutamate receptors expressed at different types of synapse is regulated in an afferent- and target-dependent fashion ${ }^{7-10}$.

Here, a method is outlined that allows analysis of the structure and receptor composition of postsynaptic membrane specializations with defined presynaptic elements and function. This approach takes advantage of presynaptic expression of recently developed light sensitive algal proteins, such as channelrhodopsin2 (ChR2), and of the FRIL technique to analyze the pattern of postsynaptic expression of $\alpha$-amino-3-hydroxy-5methyl-4-isoxazolepropionic acid (AMPA-Rs) and N-methyl-D-aspartate (NMDA-Rs) glutamate receptors. This is demonstrated at synapses formed by axons originating from the posterior thalamic-medial geniculate nuclei (PIN/MGn) onto neurons of the intercalated cell masses of the amygdala (ITC). ITC neurons are small spiny GABAergic cells organized in clusters surrounding the basolateral amygdaloid complex (BLA) ${ }^{11,}$

12. ITC neurons are known to receive excitatory inputs from BLA principal neurons and to target the central nucleus (CeA), thus functioning as an inhibitory gate for the information flow between the BLA and CeA ${ }^{12-15}$.

Recently, we demonstrated that ITC neurons located in the medio-dorsal cluster between BLA and CeA also receive direct and convergent excitatory inputs from sensory thalamic and temporal cortical regions, which are modified upon fear learning during Pavlovian auditory fear conditioning ${ }^{16}$. Fear conditioning is one of the best understood forms of associative learning in terms of brain mechanisms. In fear conditioning, an initially neutral conditioned stimulus (CS, e.g., a tone) is paired with an aversive unconditioned stimulus (US, e.g., a mild foot shock) resulting in a CS-US association and conditioned fear response ${ }^{17,18}$. Excitatory inputs from both thalamic and neocortical areas, which carry information representing the CS and US, respectively, were known to converge onto pyramidal neurons of the lateral nucleus of the amygdala (LA) and to undergo plasticity ${ }^{19}$. Our previous work revealed that sensory input information is also in parallel relayed to ITC neurons ${ }^{16}$.

As a first step towards a mechanistic molecular analysis of individual sensory input synapses onto ITC neurons, we used an adeno-associated virus (AAV) to express ChR2 tagged with the yellow fluorescent protein (YFP). The AAV was injected into the PIN/MGn and the axon terminals were identified by their expression of ChR2-YFP. We used both faces generated by the FRIL technique to assess the density of postsynaptic AMPA-Rs and NMDA-Rs at synapses formed with ITC neurons by PIN/MGn axon terminals.

\title{
Protocol
}

Procedures involving animal subjects have been approved by the Regierungspraesidium Tübingen, State of Baden-Württemberg, Germany, and by the Austrian Animal Experimentation Ethics Board, and were in accordance with the EU directive on the use of animals in research.

\section{Stereotactic Injections of AAV-ChanneIrhodopsin2-YFP}

\author{
NOTE: Stereotactic injections were carried out according to a previously published protocol ${ }^{20}$
}

1. Prepare sterile tools by heating them at $180^{\circ} \mathrm{C}$ for $1.5 \mathrm{hr}$

2. Pull sharp $(\sim 50 \mu \mathrm{m}$ diameter $)$ glass pipettes for injections using a horizontal microelectrode puller set with the following parameters: Heat $=$ Ramp value -20 , Pull $=0$, Velocity $=100$, Time $=200$, Pressure $=200$.

NOTE: Ramp value needs to be determined for each lot of purchased glass pipettes according to the instructions provided by the micropipette puller manufacturer.

3. Premix $1 \mu \mathrm{l}$ of virus solution and $0.2 \mu \mathrm{l}$ of $0.1 \%$ fast green solution (for better visibility of the solution in the glass pipette) in sterile phosphate buffer saline (PBS; $25 \mathrm{mM}, 0.9 \% \mathrm{NaCl}, \mathrm{pH} 7.4$ ). Fill the glass pipette by using a $10 \mu \mathrm{l}$ pipette and gel-fil tips. For the viral construct, use rAAVhSyn-ChR2(H134R)-eYFP (serotype 2/9).

4. Anesthetize mouse using a small animal anesthesia device ( $3 \%$ isoflurane in oxygen for induction). Use. For this study, use 3 wild-type mice aged $\sim 6$ weeks.

5. Shave head between ears and eyes and disinfect with a povidone-iodine based solution.

6. Apply an eye ointment to prevent drying of eyes during anesthesia. Subcutaneously inject mouse with analgesic (meloxicam-based, $0.1 \mathrm{ml}$ of a $5 \mathrm{mg} / \mathrm{ml}$ solution).

7. Place mouse in stereotactic frame and maintain anesthesia via a gas anesthesia device ( $2 \%$ isoflurane in oxygen for maintenance). Check anesthesia depth by lack of a limb withdrawal reflex before continuing.

8. Maintain sterile conditions as best as possible during the entire surgical procedure. Wear a disposable face-mask, a surgical gown and gloves.

9. Make a skin incision of approximately $1 \mathrm{~cm}$ on top of the head using scissors ${ }^{20}$. Gently pull skin to the side using blunt forceps, fix with clamps to expose skull surface and clean skull with $\mathrm{H}_{2} \mathrm{O}_{2}$. 
10. Mark injection sites on the skull using a fine tip permanent marker. Drill a small hole (approximately $1 \mathrm{~mm}$ in diameter) into the skull at the marked site. For unilateral PIN/MGn injection in this stud, use the following coordinates: from bregma (mm): posterior 3.0, lateral \pm 1.8 , ventral 3.8.

11. Mount filled glass pipette onto stereotactic frame connected to a pressure injection device and bring the pipette to bregma position.

12. Break off the tip of the glass pipette using fine straight tip forceps. Make sure the pipette tip is open by applying a few pressure pulses and observing extrusion of drops of virus solution.

13. Go to the desired injection coordinates and inject half of the pipette content $(\sim 0.5 \mu \mathrm{l})$ using the following settings on the pressure injection device: pressure $20 \mathrm{psi}$, average pulse length $30 \mathrm{~ms}$, average number of pulses 50 .

14. Leave pipette in place for $\sim 1 \mathrm{~min}$ before slowly $(1 \mathrm{~mm} / \mathrm{min})$ retracting it.

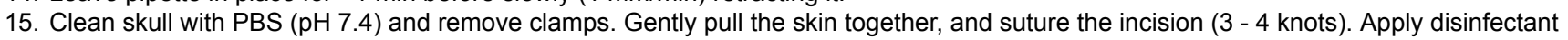
(povidone-iodine based) around the wound.

16. Stop the anesthesia and do not leave the mouse unattended until fully awake. Keep mice single-housed. Postoperatively, continue to monitor the health status; if necessary administer analgesic.

17. Keep animals for 4 weeks before brain fixation to ensure appropriate levels of viral expression.

\section{Specimen Preparation}

\section{Brain fixation}

1. Preparation of the fixative

1. For $1 \mathrm{~L}$ of fixative, weigh $10 \mathrm{~g}$ of paraformaldehyde and add it to $300 \mathrm{ml}$ of deionized $\mathrm{H}_{2} \mathrm{O}$. Heat to $55-60{ }^{\circ} \mathrm{C}$ for $\sim 10 \mathrm{~min}$ with continuous stirring.

2. Switch off the heat and add $7-8$ drops of $4 \mathrm{~N} \mathrm{NaOH}$. The solution should become clear in $\sim 10$ min.

3. Let it cool down to RT, add $150 \mathrm{ml}$ of a saturated solution of Picric acid and bring it to $500 \mathrm{ml}$ with deionized $\mathrm{H}_{2} \mathrm{O}$.

4. Add $500 \mathrm{ml}$ of $0.2 \mathrm{M}$ phosphate buffer (PB). Filter with filter paper. Adjust $\mathrm{pH}$ to 7.4 with $\mathrm{NaOH}$.

5. Cool the fixative to $6^{\circ} \mathrm{C}$, store it in dark glass bottles for not more than one day at $6^{\circ} \mathrm{C}$

\section{Transcardiac perfusion}

1. Anesthetize mice with an intraperitoneal injection of thiopental $(120 \mathrm{mg} / \mathrm{kg}$ body weight). Make sure the animal is deeply anesthetized by checking the pedal withdrawal reflex, which should be absent. Place the animal on its back on a perfusion table with the four extremities tied down.

2. Open the abdominal wall longitudinally with blunt-end scissors and make two additional cuts laterally along the caudal border of the rib cage, to expose the diaphragm. Cut away the diaphragm and cut the thoracic wall at the osteocartilageneous border on both sides. Lift the caudal end of the central slab of thoracic wall containing the sternum to expose the heart.

3. Remove the pericardium, make a small precise cut in the tip of the left ventricle to admit the cannula of the perfusion apparatus. Use a blunt cannula with an internal diameter of $0.6 \mathrm{~mm}$. Pass the cannula gently through the ventricle till the tip appears into the ascending aorta and secure the cannula with a clamp. To allow the blood and perfusates to exit from the blood stream, make a cut in the right atrium.

4. Perfuse mice transcardially using a peristaltic pump at a flow rate of $5 \mathrm{ml} / \mathrm{min}$ at first with $\mathrm{PBS}(25 \mathrm{mM}, 0.9 \% \mathrm{NaCl}, \mathrm{pH} 7.4)$ for approximately $1 \mathrm{~min}$, followed by ice-chilled fixative for $7 \mathrm{~min}$.

5. After fixation, sever the mouse head with a pair of scissors and then cut the skin through the midline from neck to nose. Remove the muscle to fully expose the skull.

6. Using sharp scissors, make a longitudinal cut through the occipital and interparietal bones starting from the foramen magnum Using fine tweezers remove these bones to expose the whole cerebellum. Then make another longitudinal cut through the parietal and frontal bones till the nasal bone and remove them with tweezers to expose the whole brain.

7. Using a spatula remove the brain without damaging it, and place it in ice-cold $0.1 \mathrm{M} \mathrm{PB}$.

\section{Sectioning and trimming of the specimens}

1. Cut a coronal block of approximately $5-6 \mathrm{~mm}$ with razor blades containing the area of interest. Glue it onto the holder of the vibroslicer with a cyanoacrylate glue. Orient the tissue block so that the neocortex faces the vibrating blade. Slice coronal sections, containing the amygdala, at $140 \mu \mathrm{m}$ with the vibroslicer (Figure 1A) in ice-cold 0.1 M PB, and collect them in a 6-well dish in the same buffer.

2. Under a stereomicroscope, trim out the region of interest (here, the medio-dorsal paracapsular cluster of the ITC, see Figure 1B) from the slice. Do this in a Petri dish coated with silicone elastomer and filled with $0.1 \mathrm{M} \mathrm{PB}$, using an ophthalmic scalpel. Make sure that the trimmed blocks fit into the hole of the spacer (approximately $1.5 \mathrm{~mm}$ ) (Figure 1B).

3. Move the trimmed blocks into cryoprotection solution ( $30 \%$ glycerol in $0.1 \mathrm{M} \mathrm{PB}) \mathrm{O} / \mathrm{N}$ at $6^{\circ} \mathrm{C}$.

\section{High Pressure Freezing}

NOTE: The FRIL consists of 6 essential steps (Figure 2): 1) Rapid freezing with high pressure (at 2,300 - 2,600 bar) of the specimen. 2) Fracturing of the specimen. The fracture plane generally follows the central hydrophobic core of frozen membranes, splitting them into two halfmembrane leaflets: a half that lies adjacent to the protoplasm (P-face) and a half that lies adjacent to the extracellular or exoplasmic space (Eface). 3) Replication of the specimen by vacuum-deposition of platinum and carbon. 4) Detergent-digestion of the tissue. 5) Immunogold labeling. 6) Analysis of the replica using a transmission electron microscope.

1. Preparation of copper carriers

NOTE: To be handled through the successive stages of the FRIL procedure, specimens need to be mounted onto metal (gold or copper) carriers. These carriers vary in size and design according to the mode of fracturing and type of machines used. Here we used copper carriers 
(Figure 1B,E) and a hinged "double replica table" (see Figure 3B), which when opened produces a tensile fracture through the frozen specimen (Figure 2). This allows to retain and replicate both sides of the fractured specimen.

1. Polish copper carriers with a tarnish remover using a sheet of chamois skin.

2. Place carriers in a glass pot and clean twice with a non-ionic detergent $(\mathrm{pH} \sim 1.5)$ in a sonicating water bath, then extensively wash in tap water followed by deionized water and then rinse twice with ethanol.

3. Sonicate the copper carriers in acetone for $15 \mathrm{~min}$.

4. Place the carriers on filter paper to dry.

5. Attach a ring of double-sided tape to a copper carrier (Figure 1C), which will serve as the holding well for the trimmed block (holding carrier).

\section{Freezing of the specimen}

NOTE: Handle liquid nitrogen with care and wearing appropriate goggles.

1. Turn on the high pressure freezing unit (Figure $\mathbf{1 F}$ ) at least $1.5 \mathrm{hr}$ before starting with the specimen freezing.

2. Start heating by pressing the "AIR HEATER" button, and bake out for $50 \mathrm{~min}$. Set air temperature to $80^{\circ} \mathrm{C}$.

3. Connect the nitrogen tank to the high pressure freezing unit and press the "NITROGEN" button to fill the inside Dewar with liquid nitrogen. The "NITROGEN LEVEL" lamp lights up. Start cooling by pressing the "Cooling" button.

4. Press the "DRIVE IN" button when the "NITROGEN LEVEL" goes out. Check that the hydraulic system moves the piston back and forth 3 times.

5. Press the "AUTO" button, and the "NITROGEN" button. As soon as "READY" lights up, the high pressure freezing unit is ready for high pressure freezing.

6. Place a trimmed block in the hole of the double-sided tape (Figure 1B) using a platinum wire loop which has been melted into a glass pipette.

7. Remove the excess of the cryoprotectant solution using filter paper or a brush.

NOTE: This procedure is also important to remove air bubbles that may form around the tissue and which could cause distortion of the tissue shape and/or ultrastructure.

8. Under a stereomicroscope, cover the holding carrier with another carrier, so that the tissue block is sandwiched between the two carriers.

9. Insert the carrier-sandwich into the specimen holder of the high pressure freezing unit (Figure 1D). Insert the specimen holder into the high pressure freezing unit (tip down) and secure it by screwing in the specimen holder.

10. Initiate the freezing cycle by pressing the "Jet-Auto" button. Working as quickly as possible, remove the specimen holder and submerge the tip with liquid nitrogen into an insulated box. Immerse the tips of two pairs of forceps in the liquid nitrogen to cool them.

11. Carefully remove the carrier-sandwich from the specimen holder and place it in a pre-chilled cryovial. Make sure that the carriers are only handled with liquid nitrogen-cooled forceps. Cryovials should be perforated to allow the nitrogen to flow out from the vial (Figure 1G).

12. Repeat steps 3.2.6 to 3.2.11 until all desired samples have been frozen. Multiple carrier-sandwiches containing the same type of sample can be stored in the same vial.

13. Store the cryovials containing the carriers in a cryotank until replication (Figure 1H). 

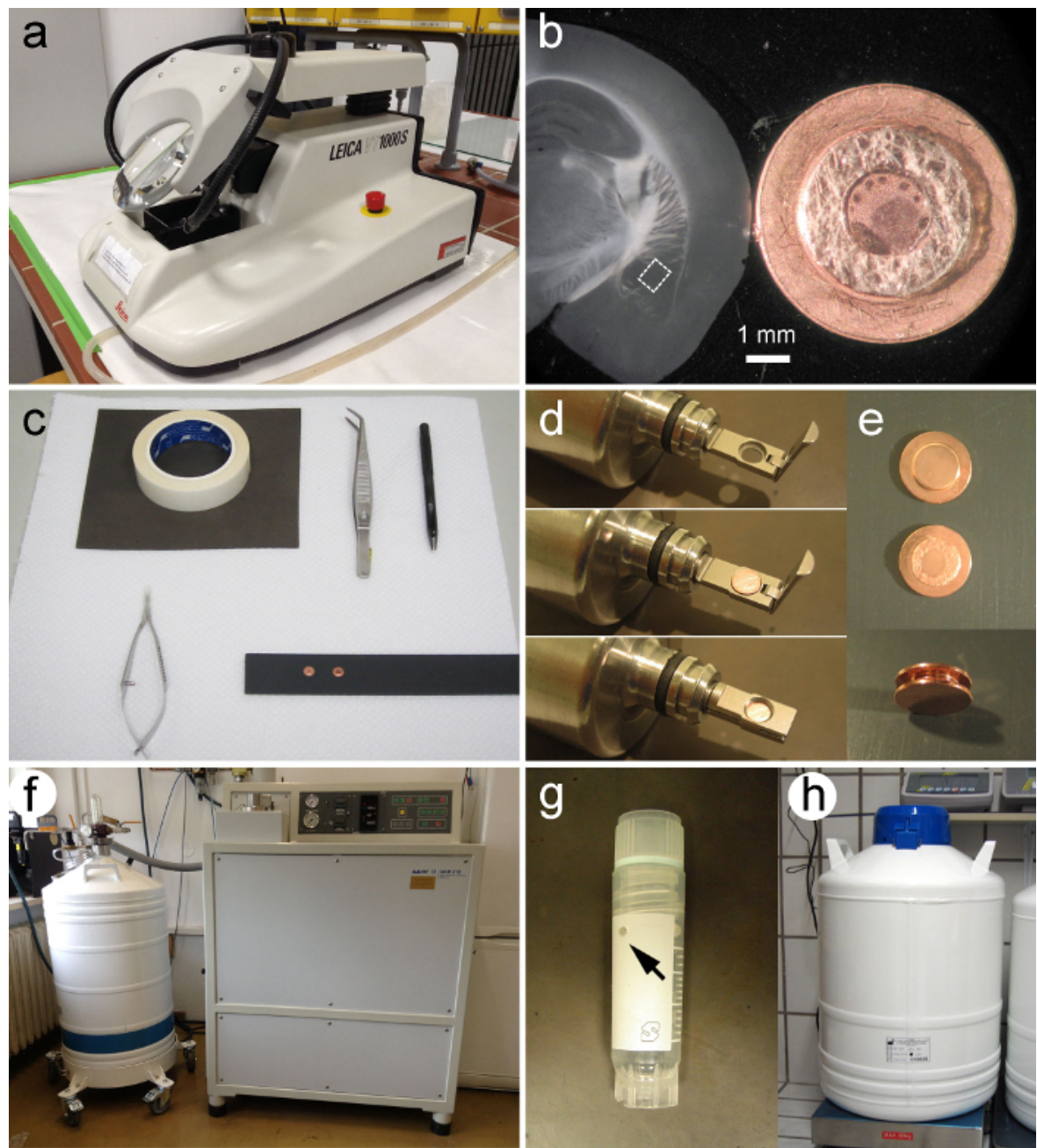

Figure 1. Tissue Preparation and High-pressure Freezing. (A) Vibroslicer used to section the tissue. (B) A resulting coronal mouse brain section containing the amygdala shown side by side with a copper carrier fitted with a ring of double-sided tape. The dashed box indicates the area of interest that contains the medial paracapsular cluster of the ITC. The diameter of the hole in the double-sided tape is approximately 1.5 $\mathrm{mm}$. (C) Tools for preparation of copper carriers. From top-left in a clockwise manner: double-sided tape, tweezers, puncher, copper carriers, and scissors. (D) Insertion of the carrier-sandwich into the specimen holder for high pressure freezing. The carrier-sandwich is placed into the hole of the specimen holder. (E) Copper carriers without and with a ring of double-sided tape and the "carrier-sandwich". (F) High pressure freezing unit with pressurized tank feeding liquid nitrogen to it. (G) A cryovial to stock the frozen tissues. Note the holes in the upper-middle position of the vial allowing nitrogen gas to flow out of the vial (arrowhead). (H) Cryotank for storing the frozen tissue. Please click here to view a larger version of this figure.

\section{Freeze-fracture and Replication}

\section{Preparation of the electron beam guns}

1. Before inserting the electron beam guns, remove the shield with the "deflector plate". Place the "setting gauge" for centering the filament into the collet chuck through the lower cathode cover. NOTE: The larger diameter end of the setting gauge is used for the carbon gun whereas the smaller diameter end is for the platinum gun.

2. Slide the new filament over the gauge until "the pressure laminae" can clamp the ends of the filament, ensuring that the filament coil does not lie at an angle.

3. Remove the setting gauge and insert the carbon rod. Fix it by tightening the collet chuck of the evaporator rod holder, ensuring that the height of the end of the rod is at the middle of the second coil from the bottom. For the platinum gun, the height of the end of the platinum rod should be at the middle of the second filament coil from the top.

4. Replace the deflector plate and insert guns into the freeze fracture unit. Clean the guns with a sand-blaster after usage. 


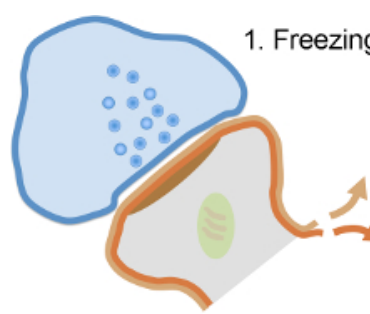

\section{Fracturing}

4. Solubilization
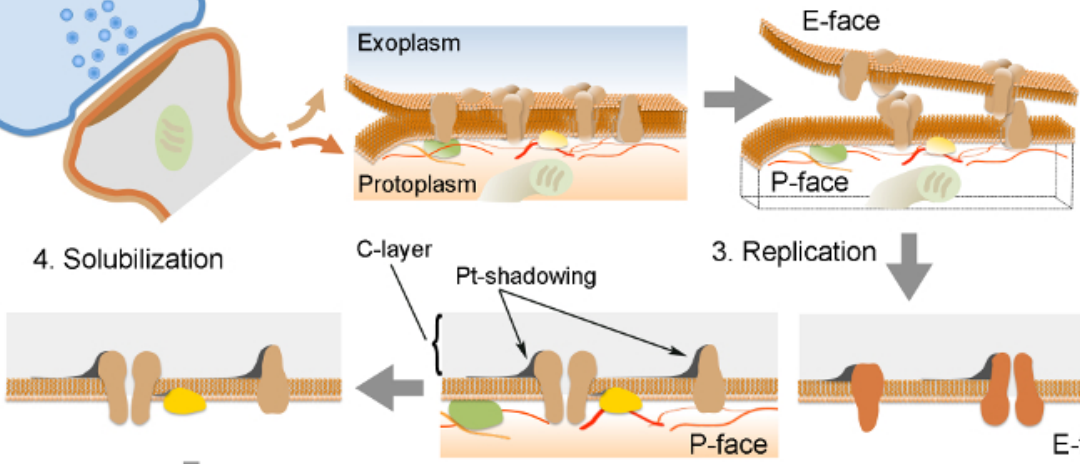

3. Replication

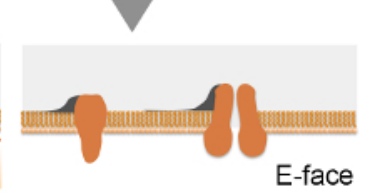

5. Labeling

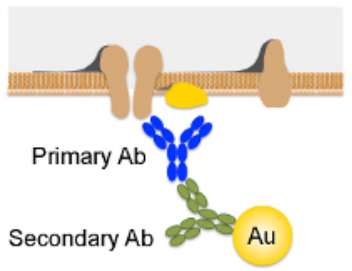

6. Analysis

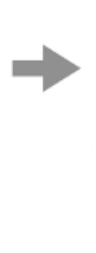

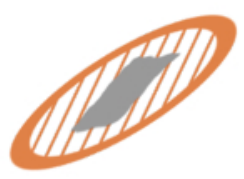

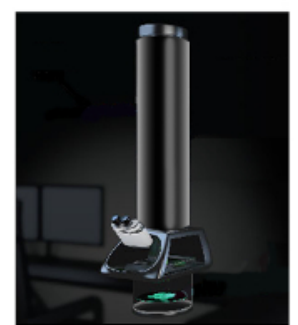

Figure 2. Illustration of the Key Steps of the FRIL Technique.

Outline of the different steps required for preparation and analysis of replica. (1) High pressure freezing of tissue. (2) Fracturing. During fracturing of frozen tissue, the lipid bilayer of plasma membranes is split into two halves at the hydrophobic interface. Proteins in the plasma membrane are allocated onto either the exoplasmic (E-face) or protoplasmic membranes (P-face). (3) Replication. The evaporation of carbon (C) traps lipids and proteins on the surface of the fractured tissue. The material is coated with $2 \mathrm{~nm}$ platinum/carbon for shadowing at a $60^{\circ}$ angle, and then with another $15 \mathrm{~nm}$ carbon layer which strengthens the structure of the replica (C-layer, Pt-shadowing). (4) Solubilization. The tissue not trapped by the replica membrane is then solubilized with SDS-solution. (5) Labeling. Proteins of interest can be visualized on replica using a complex made of specific primary antibodies (Primary Ab) and secondary antibodies (Secondary Ab) conjugated with a gold particle (Au). The use of different sizes of gold particles allows detection of more than one protein on the same replica. (6) After immunolabeling, replicas are collected onto copper mesh grids and analyzed with a transmission electron microscope at $80-100 \mathrm{kV}$. Please click here to view a larger version of this figure.

\section{Set up of freeze fracture unit}

1. Switch on the freeze fracture unit (Figure 3A) by turning MAINS to 1. For a detailed description of the freeze-fracture and replication procedures, see operating instructions provided by the manufacturer.

2. Prior to cooling the freeze-fracture device, bake out the entire cooling system of the unit with warm air. Press the "Thawing" button in the MTC 010 device (temperature control unit) (Figure 3A) and let the bake out process run for 45 min.

3. Activate the vacuum station. The freeze fracture unit usually operates in a vacuum range of $\sim 10^{-6}-10^{-7}$ mbar.

4. Fill nitrogen tank and connect it to the freeze fracture unit. Check that the valve holder is dry and also clean the entry of the tank before insertion of the valve holder (humidity can interfere with vacuum and indication of $\mathrm{N}_{2}$ filling of the tank).

5. Start cooling by setting the temperature to $-115^{\circ} \mathrm{C}$. Cooling takes about $45 \mathrm{~min}$.

6. Insert the electron beam guns and adjust current and voltage to reach the following parameters for evaporation: Carbon gun: rotation on, position $90^{\circ}$, rate of carbon accumulation $0.1-0.2 \mathrm{~nm} / \mathrm{sec}$ Carbon-platinum gun: rotation off, position $60^{\circ}$, rate of accumulation $0.06-0.1 \mathrm{~nm} / \mathrm{sec}$ NOTE: If a gun is used for the first time after exchange of the carbon or platinum rod, degas for 3 min before usage.

\section{Fracturing and replication}

1. Insert frozen carrier-sandwiches into double replica table making sure all manipulations are done in liquid nitrogen.

2. Transfer double replica table to a Dewar vessel and fix it to the specimen stage receiver at an angle of $45^{\circ}$. The liquid nitrogen level should always be above the double replica table.

3. Pick up the double replica table with the table manipulator and insert it into the freeze fracture unit onto the cold stage. Wait approximately $20 \mathrm{~min}$ to allow the temperature of the double replica table to adjust to $-115^{\circ} \mathrm{C}$.

4. Check that the vacuum is below $10^{-6} \mathrm{mbar}$ and the temperature is $-115^{\circ} \mathrm{C}$.

5. Fracture the tissue by manual counter clockwise rotation of the wheel connected to the shroud placed above the double replica table. When the shroud turns, it forces the double replica table to open, fracturing the tissue.

6. Press the "High tension" button in the EVM 030 device (electron beam evaporation control unit) of the freeze fracture unit (Figure 3A).

7. Replicate the exposed surfaces of the fractured tissue (Figure $3 \mathrm{C}$ ) by evaporation of carbon (rotating) by means of an electron beam gun positioned at a $90^{\circ}$ angle to a thickness of $5 \mathrm{~nm}$, followed by a unidirectional shadowing with platinum-carbon at a $60^{\circ}$ angle to a thickness of $2 \mathrm{~nm}$. Finally, apply a $15 \mathrm{~nm}$ thick layer of carbon from a $90^{\circ}$ angle (rotating).

8. Use the following parameters for evaporation:

1st carbon: rotation on, position $90^{\circ}$; speed $0.1-0.2 \mathrm{~nm} / \mathrm{sec} ; 5 \mathrm{~nm}$

2nd carbon-platinum: position $60^{\circ}$; speed $0.06-0.1 \mathrm{~nm} / \mathrm{sec} ; 2 \mathrm{~nm}$ 
3rd carbon: rotation on, position $90^{\circ}$; speed $0.3-0.5 \mathrm{~nm} / \mathrm{sec} ; 15 \mathrm{~nm}$

9. Remove the replicated specimens from the freeze fracture unit and transfer them to a ceramic 12-well plate (Figure 4A) filled with TBS (Tris buffered saline, $\mathrm{pH} 7.4$ ).

10. Using a platinum loop wire rod, remove the replicated tissue from the specimen carrier (Figure 4A).

11. Repeat steps 4.3.1 to 4.3.10 until all samples have been replicated.

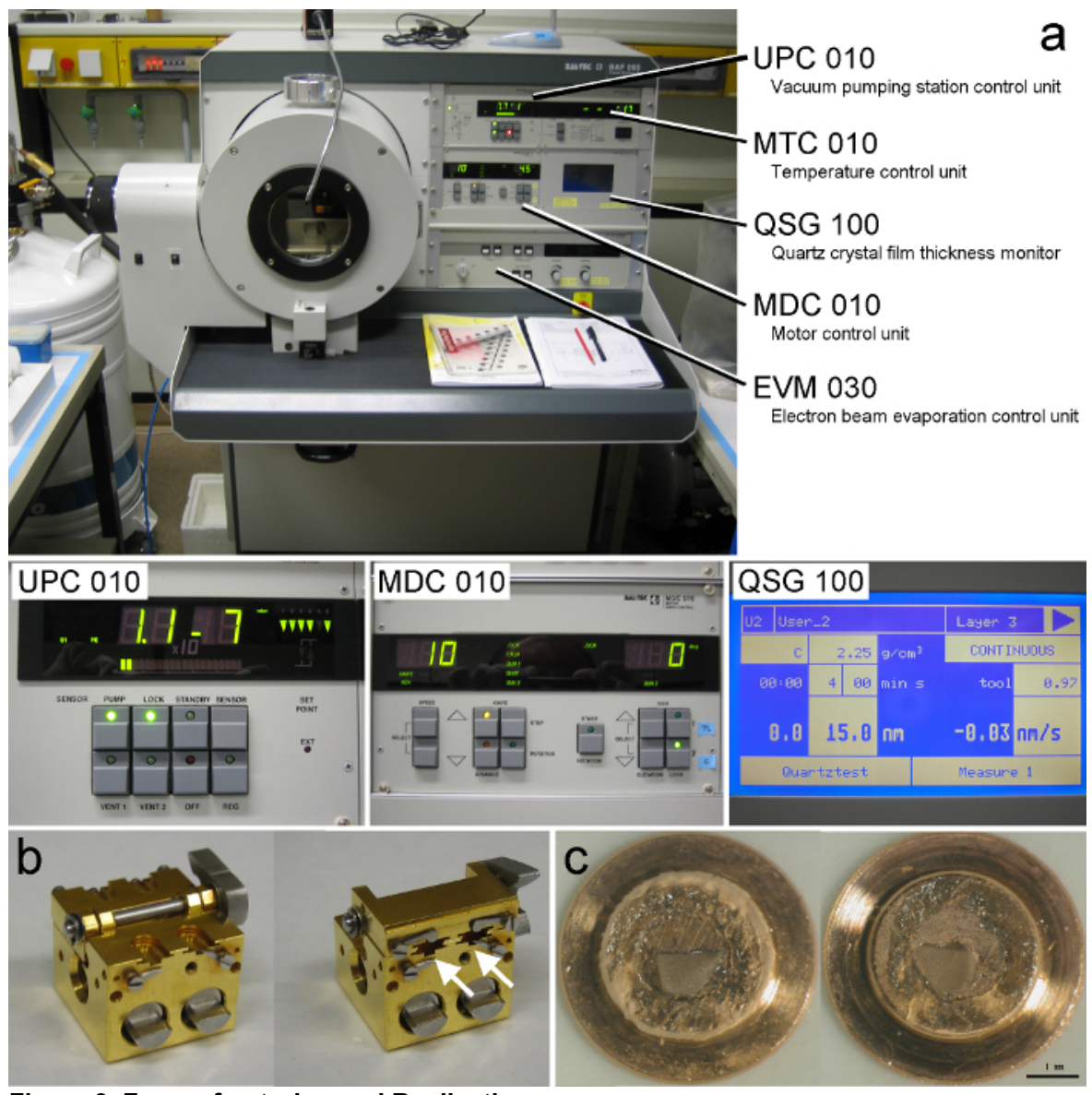

Figure 3. Freeze-fracturing and Replication.

(A) The freeze fracture unit. The machine contains several control units and a monitor. Specimens are introduced into the chamber through a port on the left side of the chamber. A pressurized liquid nitrogen container is connected to the freeze fracture unit to cool the stage. Images below show enlarged views of two of the units (UPC 010 and MDC 010) and the monitor displaying parameters during evaporation of the second carbon layer. (B) Opened (left) and closed (right) views of the double replica table. The "carrier-sandwiches" are inserted into the slots of the table (indicated by arrows). The small arms prevent "carrier-sandwiches" from falling out during manipulation. (C) A fractured and replicated sample. Replicas appear as thin black films on top of the fractured tissue. Please click here to view a larger version of this figure.

\section{SDS-digestion of the replica}

1. Transfer replica to a $4 \mathrm{ml}$ glass vial filled with $1 \mathrm{ml}$ of SDS-digestion buffer (2.5\% Sodium Lauryl Sulfate, $20 \%$ sucrose in $15 \mathrm{mM}$ Tris, $\mathrm{pH}$ 8.3). Digest for $18 \mathrm{hr}$ at $80^{\circ} \mathrm{C}$ with shaking ( $\left.45 \mathrm{stroke} / \mathrm{min}\right)$.

2. Transfer replicas to a new tube filled with SDS-digestion buffer and store at RT.

\section{Immunolabeling}

NOTE: All incubations are performed at RT with gentle shaking, except for incubations with antibodies.

1. Wash the replica for $10 \mathrm{~min}$ in fresh SDS-digestion buffer.

2. Wash the replica once with $2.5 \%$ BSA (bovine serum albumin) in TBS for 5 min, and then $3 \times 10$ min with $0.1 \%$ BSA in TBS.

3. Block non-specific binding sites in TBS with $5 \%$ BSA for $1 \mathrm{hr}$.

4. Apply primary antibodies diluted in $2 \%$ BSA-TBS. Perform incubations in a $30 \mu \mathrm{l}$ drop (Figure $4 \mathrm{~B}$ ) in a humid chamber at $15{ }^{\circ} \mathrm{C}$ for 72 hr (Figure 4C).

1. For this study, process both replicas from the fractured tissue. Incubate one replica with a guinea pig polyclonal antibody raised against the amino acids 717 - 754 of the mouse GluR1 common to all AMPA-R subunits (dilution: 1:200) or a mouse monoclonal antibody raised against a recombinant fusion protein covering amino acids 660 - 811 of the NR1 subunit of the NMDA-R (dilution: 1:500), and a rabbit polyclonal antibody raised against the green fluorescent protein (dilution: 1:300). 
2. Incubate the other replica with a rabbit polyclonal antibody raised against a synthetic peptide corresponding to amino acids $384-398$ of the rat $\mu$-opioid receptor (dilution: 1:500).

5. Wash in TBS with $0.05 \%$ BSA ( $3 \times 5 \mathrm{~min}$.).

6. Apply secondary antibodies. For this study use gold ( $5 \mathrm{~nm}$ for ionotropic glutamate receptors, 10 for $\mu$-opioid receptors and/or $15 \mathrm{~nm}$ for ChR2-YFP) conjugated antibodies diluted in TBS with $2 \%$ BSA. Dilute secondary antibodies $1: 30$ and incubate in a $30 \mu \mathrm{drop}$ at $15{ }^{\circ} \mathrm{C}$ O/N.

7. Wash $3 \times 5$ min in $0.05 \%$ BSA-TBS at RT.

8. Wash $2 \times 5 \mathrm{~min}$ in ultrapure water.

9. Mount replica on formvar-coated 100-line parallel bar grid (Figure 4D).

\section{Replica Analysis}

1. Image replicas with a transmission electron microscope (TEM) at 80 or $100 \mathrm{kV}$. Acquire digital images through a CCD (charged coupled device) camera.

2. Offline, find corresponding regions on images from both replicas using landmarks (Figure 4E). Analyze digital images using Image $\mathrm{J}$. Determine postsynaptic area and the number of immunogold-labeled particles directed against the receptor analyzed.
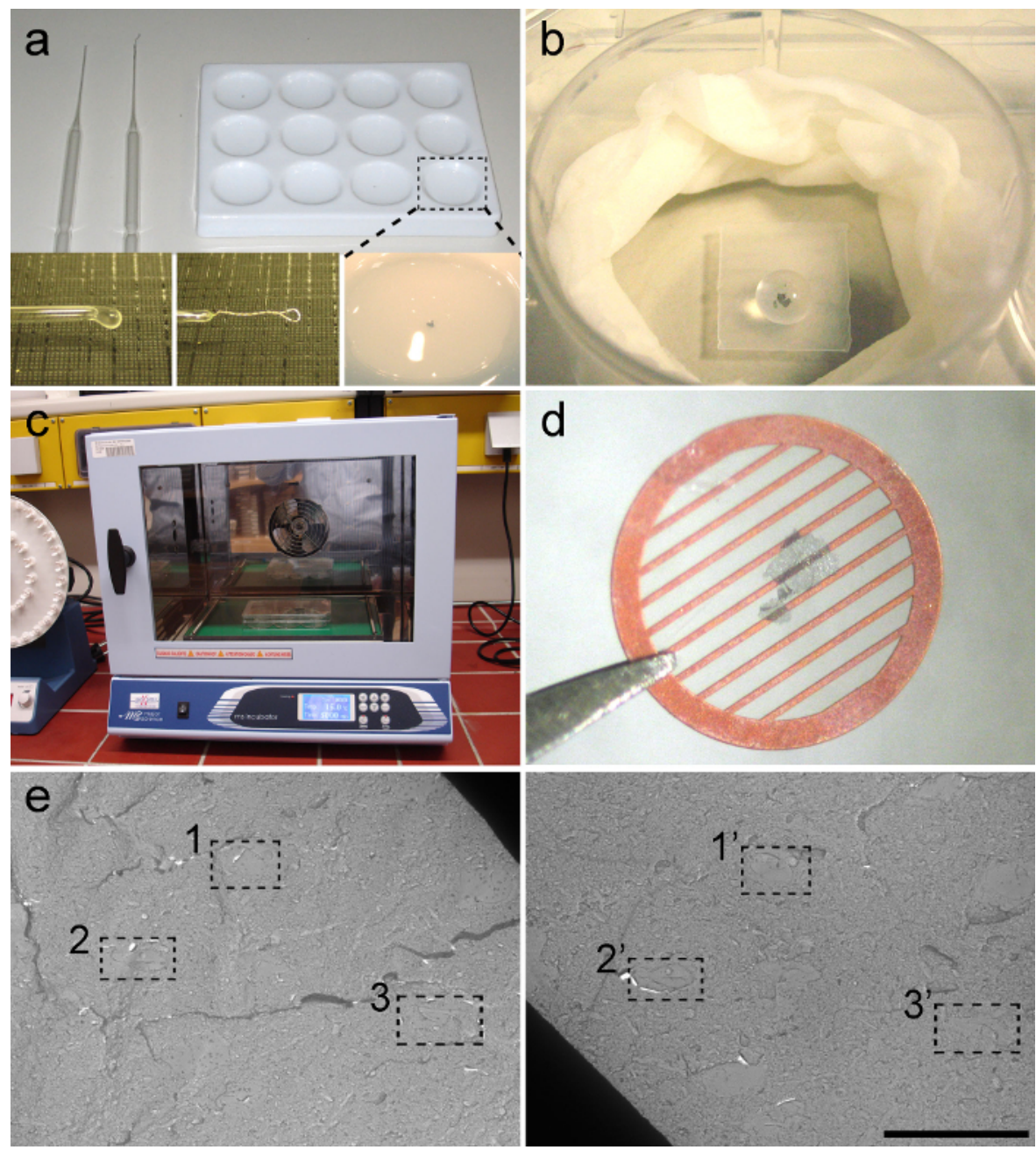

\section{Figure 4. Immunolabeling of Replica.}

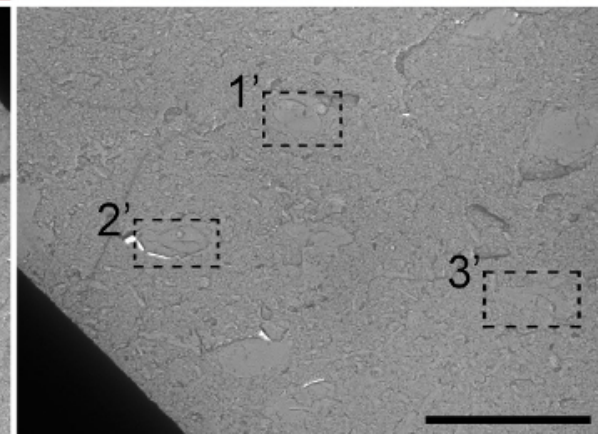

(A) Tools for manipulating and washing the replicas. A ceramic 12-well plate (top right) and 2 types of glass pipettes (top left). The glass pipette with round tip (bottom left) is used to transfer replica, and the pipette with platinum rod (bottom centre) is used to unfold replicas. A replica in washing buffer (bottom right). (B) Immunolabeling of replicas is carried out in drops (30 $\mu$ l) placed on a small piece of parafilm in a well of a tissue culture 6-well plate. Note that a replica is covered by a the drop of buffer containing antibodies. To prevent evaporation, a moistened piece of tissue paper is fitted around the inner edge of the well. (C) Incubator for the immunolabeling step. Incubations are carried out at $15{ }^{\circ} \mathrm{C}$. (D) A replica mounted on a formvar-coated 100-line parallel bar grid. (E) Low magnification micrographs from a pair of replicas. The dotted squares indicate three typical landmarks to identify a location in the corresponding replicas. Scale bar: $10 \mu \mathrm{m}$. All data are shown as mean \pm s.e.m. Please click here to view a larger version of this figure. 


\section{Representative Results}

The FRIL technique, when combined with expression of optogenetic actuators of microbial origin ${ }^{21}$, i.e., channels integrated in the plasma membrane and effectively transported anterogradely along axons, allows to examine quantitatively the postsynaptic expression of AMPA-Rs and NMDA-Rs at a defined subgroup of synapses. This is shown here for axons originating from distinct thalamic nuclei (e.g., PIN/MGn) onto ITC neurons in the amygdala. This approach enables a molecular analysis of individual sensory input synapses onto ITC neurons, a group of cells that have been refractory to a detailed anatomical and molecular characterization so far.

Four weeks after stereotactic injection of the rAAV-ChR2-YFP into the PIN/MGn, ChR2-YFP-positive axons densely innervated the LA, the amygdalostriatal transition area (Astria) and the medial paracapsular ITC cluster in the amygdala, a pattern fully consistent with previous tracing studies ${ }^{16,22}$. We also detected intense gold immunolabeling for the ChR2-YFP on the P-face of axons and terminals in freeze-fracture replica from rAAV-ChR2-YFP-injected mice (Figure 5A), but not in replicas from non-injected mice. The postsynaptic membrane specialization of glutamatergic synapses in a replica can be observed as a cluster of intramembrane particles (IMPs) on the E-face of the plasma membrane ${ }^{2}, 23$, and is often accompanied by the P-face of its presynaptic plasma membrane ${ }^{7}$ (Figure 5B-C). These features allowed to identify the postsynaptic specialization of glutamatergic synapses formed by PIN/MGn axon terminals (Figure 5 and 6). We labeled AMPA-Rs with an antibody that recognizes all four subunits (GluA1-4), whereas NMDA-Rs were detected using an antibody against the essential NR1 subunit.

Because of the lack of tools to detect on the same replica whether these synapses were made with dendritic spines or shafts of ITC neurons, we labeled the corresponding replica face for $\mu$-opioid receptors, as ITC neurons express postsynaptically high levels of these receptors ${ }^{24}$. This required the identification of the same postsynaptic profiles in the two replicas (Figure 5C-F and Figure 6A-D) using a strategy that employs landmarks (Figure 4E). 

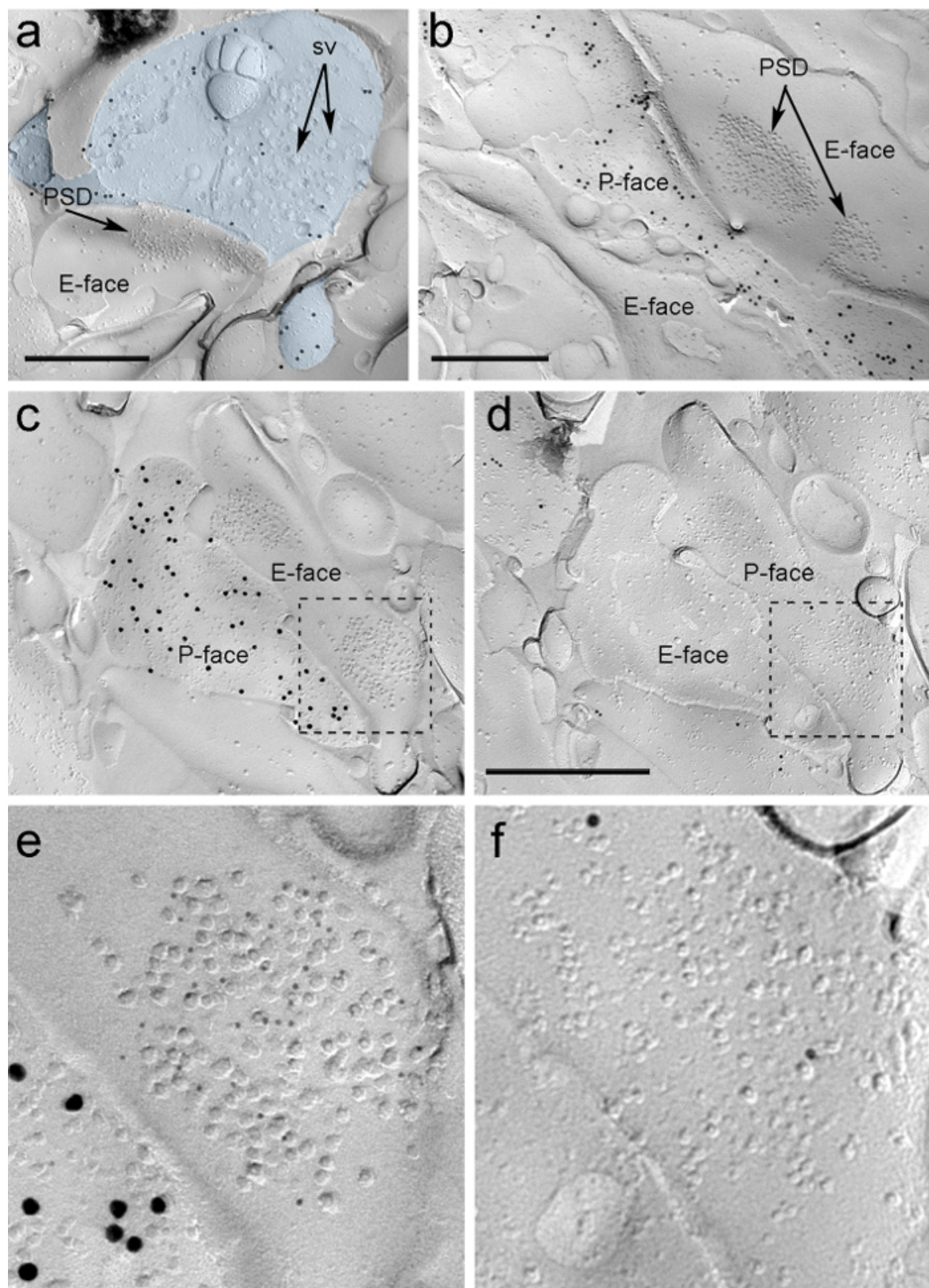

Figure 5. Detection of ChR2-YFP and lonotropic Glutamate Receptors on Replica by Immunogold Particles. (A) A cross-fractured axon terminal (light blue) and small portions of its P-face labeled with $15 \mathrm{~nm}$ gold particles detecting ChR2-YFP. Within the terminal, the membrane of numerous synaptic vesicles can be observed (sv). Note the specificity of the immunolabeling in large part restricted to the plasma membrane. Labeling for ChR2 identifies the terminal as originating from the PIN/MGn. The terminal forms an asymmetric synapse with a spine. The postsynaptic membrane specialization (PSD) on the E-face shows a characteristic cluster of intramembrane particles and is labeled with $5 \mathrm{~nm}$ gold particles revealing AMPA-Rs. (B) The P-face of a ChR2-expressing axon (labeled with $15 \mathrm{~nm}$ gold particles) is shown bordering two dendrites, one of them possessing two PSDs labeled with $5 \mathrm{~nm}$ gold particles revealing NMDA-Rs. (C-D) Opposite faces of the pre- and postsynaptic membranes of a PIN/MGn-ITC synapse. (C) The P-face of the terminal expresses ChR2 (labeled with $15 \mathrm{~nm}$ gold particles) and extends over the E-face of two dendritic shafts, one of them containing two PSDs labeled for AMPA-Rs (5 nm gold particles). (D) The corresponding P-face of the two dendrites is labeled for $\mu$-opioid receptors (10 nm gold particles). (E-F) Enlarged views of the areas outlined by the dashed lines. Scale bars: $500 \mathrm{~nm}$. Please click here to view a larger version of this figure.

Immunoparticles for AMPA-Rs in the PIN/MGn-ITC synapses were found all over the IMP cluster, suggesting a homogeneous distribution within the postsynaptic specialization (Figure 5E). A significantly higher (unpaired t-test $p<0.018$ ) density of AMPA-R labeling was observed in PIN/ MGn synapses onto ITC spines $\left(715 \pm 38\right.$ gold particles $\left./ \mu \mathrm{m}^{2}, \mathrm{n}=32\right)$ compared to synapses onto ITC dendrites $\left(590 \pm 44 \mathrm{gold}\right.$ particles $/ \mu \mathrm{m}^{2}, \mathrm{n}=$ 32). Overall, the density of AMPA-Rs in PIN/MGn-ITC synapses showed a relatively low variance (Coefficient of variance, $\mathrm{CV}=0.37$ ) consistent with a homogeneous distribution. 
Immunoparticles for NMDA-Rs in the PIN/MGn-ITC synapses were often observed unevenly distributed within postsynaptic IMP clusters (Figure 5B). The density of NMDA-R labeling was similar (unpaired t-test $p=0.39$ ) between PIN/MGn synapses onto ITC spines (1070 \pm 153 gold particles/ $\left.\mu \mathrm{m}^{2}, \mathrm{n}=8\right)$ and ITC dendrites ( $812 \pm 183$ gold particles $\left./ \mu \mathrm{m}^{2}, \mathrm{n}=9\right)$. Unlike what was observed for AMPA-Rs, the density of NMDA-Rs in PIN/MGnITC synapses was highly variable $(\mathrm{CV}=0.54)$.
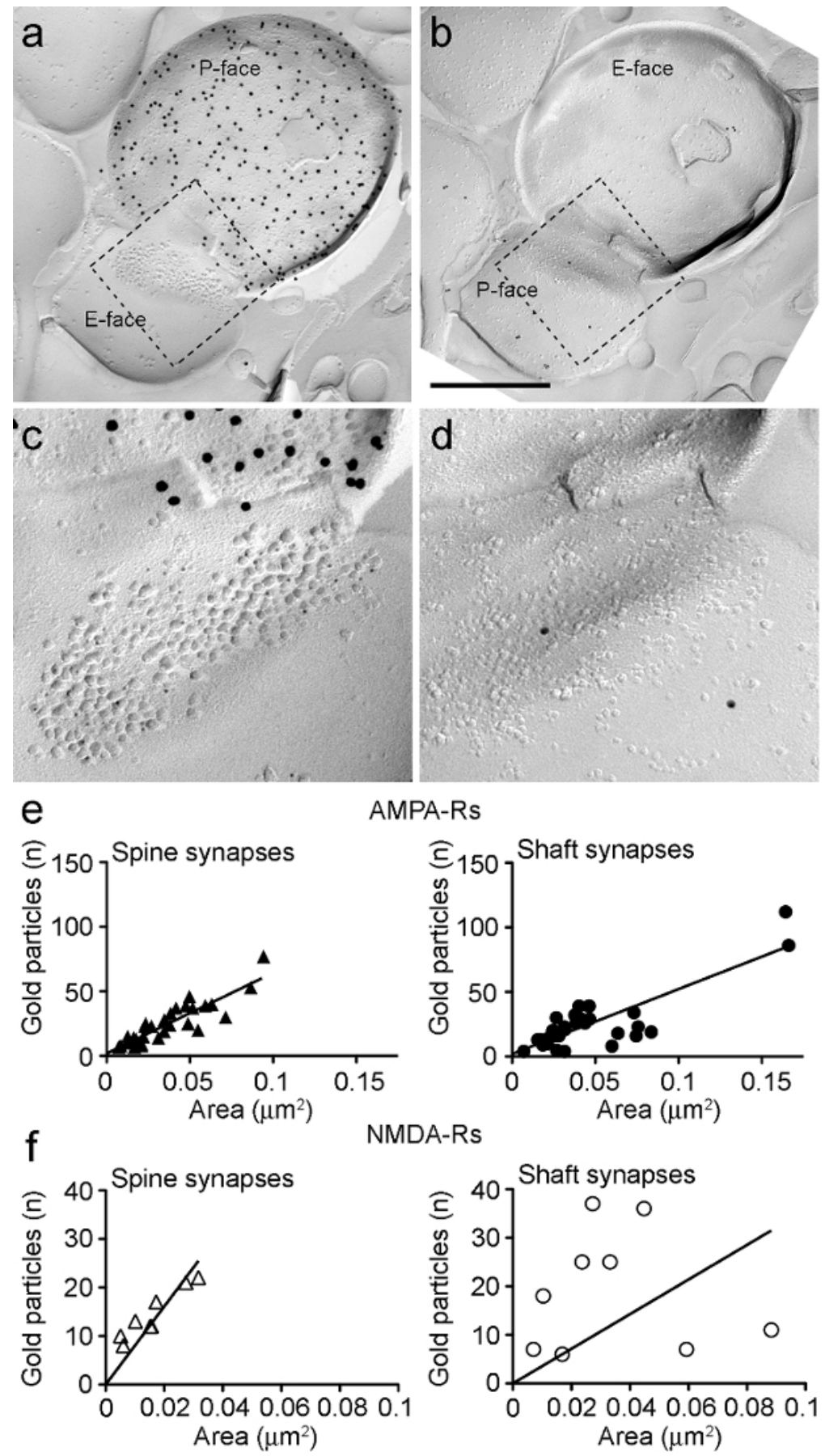

AMPA-Rs
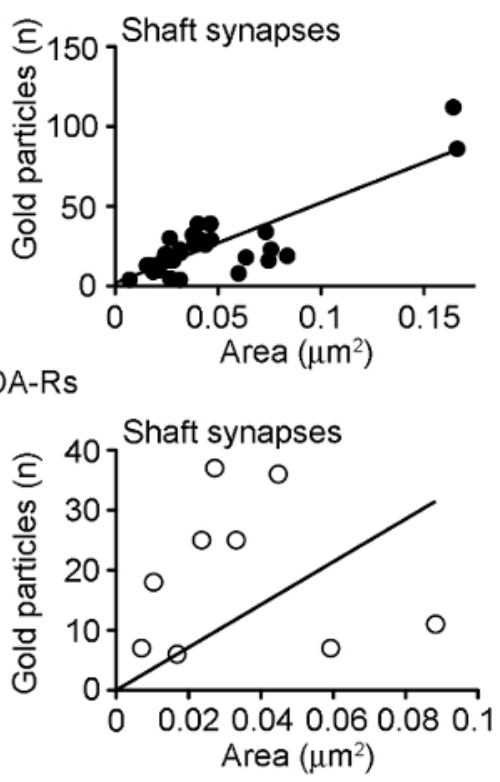

Figure 6. AMPA-Rs and NMDA-Rs Immunolabeling at Identified PIN/MGn-ITC Synapses.

(A-B) Opposite faces of the pre- and postsynaptic membranes of a PIN/MGn-ITC synapse made onto a dendritic spine in which the P-face of the terminal expresses ChR2 (labeled with $15 \mathrm{~nm}$ gold particles) and the PSD onto a dendritic spine is labeled for AMPA-Rs (5 nm gold particles). (C-D) Enlarged views of the areas outlined by the dashed line. These areas have been rotated approximately $45^{\circ}$ anticlockwise to allow a better view of the PSD. (E) Scatterplots of the number of AMPA-R particles versus synaptic area in ITC spines and dendrites. In both structures, a positive correlation has been observed. (F) Scatterplots of the number of NMDA-R particles against synaptic area in ITC spines and dendrites. A significant positive correlation was detected only in dendritic spines. Scale bars: $500 \mathrm{~nm}$. Please click here to view a larger version of this figure. 
Because the P-face of the presynaptic plasma membrane often overlaid in part the postsynaptic IMP cluster, we could estimate the synaptic area of only $30 \%$ of the synapses (spines: mean area $0.032 \mu \mathrm{m}^{2}$, range: 0.007 to $0.063 \mu \mathrm{m}^{2}, \mathrm{n}=8$; dendrites: $0.047 \mu \mathrm{m}^{2}$, range: 0.024 to $0.166 \mu \mathrm{m}^{2}$, $\mathrm{n}=11$ ). These were in a similar range as previously analyzed telencephalic glutamatergic synapses ${ }^{25}$.

In both spines and dendrites, the number of gold immunoparticles for AMPA-Rs in individual synapses was positively correlated with the synaptic area (Spearman, spines: $r=0.88$, dendrites: $r=0.60, p<0.0001$ ) (Figure 6E). Conversely, the number of gold immunoparticles for NMDA-Rs was found to correlate with the synaptic area in spines (Spearman, spines: $r=0.90, p<0.002)$ but not in dendrites $(r=0.21, p=0.29)(F i g u r e 6 F)$.

\section{Discussion}

Freeze-fracture electron microscopy has been a major technique in ultrastructural research for over 40 years. However, the lack of effective means to study the molecular composition of membranes produced a significant decline in its use. Recently, there has been a major revival in freeze-fracture electron microscopy due to the development of effective ways to reveal integral membrane proteins by immunogold labeling ${ }^{1,2}$, namely the FRIL technique.

The FRIL technique possesses several advantages over other immunogold ultrastructural methods. First, proteins are readily accessible to antibodies increasing the sensitivity. Second, exposure of large portion of plasma membrane specializations, such as the postsynaptic membrane, on the two-dimensional surface of the replica allows the inspection of the spatial distribution and physical contiguity of molecules of interest without laborious and time-consuming reconstruction of serial ultrathin sections. Third, the availability of both halves of the plasma membrane increases the number of proteins that can be labeled for each individual structure, provided suitable antibodies are available. Upon fracturing, the hydrophobic face of the split membrane is coated with carbon-platinum that entrenches protein domains remaining on the fractured surface. This prevents access of antibodies to antigens in these domains. For instance on the P-face of a replica only epitopes facing the protoplasmic space can be detected by antibodies, whereas on the E-face only epitopes facing the exoplasmic space can be bound by antibodies (see Figure 2).

On the other hand, the FRIL technique also suffers from certain limitations ${ }^{2}$. As fractures occur randomly, it might be difficult to target specific cells or structures. This can also lead to a sampling bias, e.g., in synapse collection, given the different probability of fracturing along the membrane of structures with different curvature (e.g., spines versus shafts). Moreover, the allocation of membrane proteins to one of the two faces is unpredictable. Therefore, the distribution of a protein to the P-face or E-face, particularly for quantitative studies, should be carefully examined using antibodies reactive to intracellular and extracellular domains. Finally, the identification in the replica of certain structures, such as presynaptic axon terminals, can be difficult when based only on morphological features. However, the use of specific antibodies for marker proteins or the transduction of tagged integral membrane proteins or channels using viral vectors offers additional tools to facilitate identification of the fractured membranes. For example, this study took advantage of the transduction of ChR2-YFP in thalamic neurons to identify their axonal efferents in the amygdala or the labeling for $\mu$-opioid receptors to reveal postsynaptic membranes of ITC neurons.

In order to perform the FRIL technique successfully, particular care should be taken concerning tissue fixation. Strong tissue fixation (> $2 \%$ paraformaldehyde) can result in a high rate of cross-fractures and a decrease in labeling sensitivity. On the other hand, weak fixations make the tissue handling and preparation (e.g., cutting of sections) difficult. It is also important to control that the thickness of the trimmed blocks matches the thickness of the double-sided tape. If the thickness of the specimen is lower than that of the tape, the surfaces of the tissue might not attach to the surface of the two metal carriers, consequently the frozen specimen is not fractured. If the tissue is thicker, it will be compressed with inevitable structural distortions when the sandwich of the two copper carriers is made. The temperature at which the specimen is fractured (in this protocol, $-115^{\circ} \mathrm{C}$ ) plays also an important role on the structure of the replica. Higher temperatures may produce a higher rate of artifacts such as condensation of water vapor on the surface of the tissue prior or during evaporation. Lower temperatures $\left(<-125^{\circ} \mathrm{C}\right)$ may increase the risk of splitting off of material during fracturing. This material may fall onto the surface of the specimen or stay connected to it. These flakes of material are also coated and contrasted producing dark spots on the image. Fracturing at lower temperatures can also affect the frequency of cross-fractures particularly for small fine structures such as dendritic spines. A further critical step in the preparation of replicas is the detergentdigestion. If the digestion is incomplete, the undigested tissue appears as dark patches on the replica, confounding the analysis of the structure at the TEM. Moreover, the undigested tissue can non-specifically trap or bind antibodies, increasing the background labeling. On the other hand, the use of detergents for tissue digestion can denature the molecules associated to the replica altering their secondary and tertiary structures. Therefore, for certain antigens it might be necessary to gradually dilute the concentration of SDS with additional washing steps.

For immunolabeling, the availability of different sizes of gold particle conjugated to secondary antibodies allows to detect at the same time, but only qualitatively, multiple proteins, even in specific microdomains of the plasma membrane, such as the postsynaptic specialization. However, due to steric hindrance, quantitative studies are generally limited to the detection of just one molecule. The size of the gold particle can also affect the labeling efficacy.

For the interpretation of the labeling in FRIL, it should be kept in mind that the immunogold particle can be located anywhere within a hemisphere with a radius of $20-25 \mathrm{~nm}$ from the antigen due to the flexible complex formed by the primary and secondary antibody ${ }^{26}$. For further information on the theory and practice of FRIL and related techniques, we refer the reader also to other methodological articles 27,28

The FRIL technique has been recently used for high-resolution quantitative analyses of glutamate receptor localization in diverse synapse populations ${ }^{29,30}$. Moreover, the detection sensitivity of the FRIL technique for AMPA-Rs was estimated as high as one immunogold particle per one functional AMPA-R channel ${ }^{29}$. Thus, this approach is overall very useful to quantify and analyze the pattern of postsynaptic expression of AMPA-Rs and NMDA-Rs at central synapses. Here, we demonstrated its applicability at PIN/MGn-ITC synapses, a site most likely important for relaying US information during fear conditioning. Using an antibody raised against the highly conserved extracellular amino acid residues of the AMPA receptor subunits GluA1-GluA4, we found an even distribution of gold particles within IMP clusters corresponding to postsynaptic membrane specializations. The density of AMPA-Rs in ITC spines was significantly higher compared to shaft synapses targeted by PIN/ MGn thalamic afferents. At both spine and shaft synapses, a positive correlation between labeling for AMPA-Rs and postsynaptic area was detected, a feature common to other glutamatergic synapses ${ }^{25}$. The low variance in density of AMPA-Rs in PIN/MGn-ITC synapses indicates a homogeneous distribution similar to other synapses formed by thalamic efferents ${ }^{7}$, but different from cortical synapses ${ }^{25}$. Conversely, the 
density of NMDA-Rs was more variable and did not differ between spine and shaft synapses suggesting a different regulation than AMPA-Rs. In the future, the high reproducibility of the FRIL technique will not only allow to assess the basal molecular composition of central synapses but may facilitate detection of changes in ionotropic glutamate receptor numbers and subsynaptic distribution after fear learning, complementing exvivo recordings of pre- and postsynaptic properties of these inputs.

In conclusion, this approach could be used by other investigators to gain insights into structure-function relationships of input-specific excitatory synapses in many other neural circuits in which disentangling the origin of the inputs and the nature and composition of postsynaptic elements is crucial but problematic.

\section{Disclosures}

The authors declare that they have no competing financial interests.

\section{Acknowledgements}

Funding was provided by the Austrian Science Fund FWF grant No. P-22969-B11 to F. Ferraguti, and by the Charitable Hertie Foundation and the Werner Reichardt Centre for Integrative Neuroscience and by the DFG (CIN-Exc. 307) to I. Ehrlich.

\section{References}

1. Fujimoto, K. SDS-digested freeze-fracture replica labeling electron microscopy to study the two-dimensional distribution of integral membrane proteins and phospholipids in biomembranes: practical procedure, interpretation and application. Histochem Cell Biol. 107 (2), 87-96 (1997).

2. Masugi-Tokita, M., \& Shigemoto, R. High-resolution quantitative visualization of glutamate and GABA receptors at central synapses. Curr Opin Neurobiol. 17 (3), 387-393 (2007).

3. Rash, J.E., \& Yasumura, T. Direct immunogold labeling of connexins and aquaporin-4 in freeze-fracture replicas of liver, brain, and spinal cord: factors limiting quantitative analysis. Cell Tissue Res. 296 (2), 307-321 (1999).

4. Emes, R.D., \& Grant, S.G. Evolution of synapse complexity and diversity. Annu Rev Neurosci. 35, 111-131 (2012).

5. Matsuzaki, M. et al. Dendritic spine geometry is critical for AMPA receptor expression in hippocampal CA1 pyramidal neurons. Nat Neurosci. 4 (11) 1086-1092 (2001).

6. Rollenhagen, A., \& Lübke, J.H. The morphology of excitatory central synapses: from structure to function. Cell Tissue Res. 326 (2), $221-237$ (2006).

7. Tarusawa, E. et al. Input-specific intrasynaptic arrangements of ionotropic glutamate receptors and their impact on postsynaptic responses. $J$ Neurosci. 29 (41), 12896-12908 (2009).

8. Nusser, Z. et al. Cell type and pathway dependence of synaptic AMPA receptor number and variability in the hippocampus. Neuron. 21 (3), 545-559 (1998).

9. Nyìri, G., Stephenson, F.A., Freund T.F., \& Somogyi, P. Large variability in synaptic N-methyl-D-aspartate receptor density on interneurons and a comparison with pyramidal-cell spines in the rat hippocampus. Neuroscience. 119 (2), 347-363 (2003).

10. Nicholson, D.A., \& Geinisman, Y. Axospinous synaptic subtype-specific differences in structure, size, ionotropic receptor expression, and connectivity in apical dendritic regions of rat hippocampal CA1 pyramidal neurons. J Comp Neurol. 512 (3), 399-418 (2009).

11. Millhouse, O.E. The intercalated cells of the amygdala. J Comp Neurol. 247 (2), 246-271 (1986).

12. Busti, D. et al. Different fear states engage distinct networks within the intercalated cell clusters of the amygdala. J Neurosci. 31 (13), 5131-5144 (2011).

13. Amano, T., Unal, C.T., \& Paré, D. Synaptic correlates of fear extinction in the amygdala. Nat Neurosci. 13 (4), $489-494$ (2010).

14. Duvarci, S., \& and Pare, D. Amygdala microcircuits controlling learned fear. Neuron. 82 (5), 966-980 (2014).

15. Jüngling, K. et al. Neuropeptide S-mediated control of fear expression and extinction: role of intercalated GABAergic neurons in the amygdala. Neuron. 59 (2), 298-310 (2008).

16. Asede, D., Bosch, D., Lüthi, A. Ferraguti, F., \& Ehrlich, I. Sensory inputs to intercalated cells provide fear-learning modulated inhibition to the basolateral amygdala. Neuron. 86 (2), 541-554 (2015).

17. Maren, S. Neurobiology of Pavlovian fear conditioning. Annu Rev Neurosci. 24, 897-931 (2001).

18. Pape, H.C. and Pare, D. Plastic synaptic networks of the amygdala for the acquisition, expression, and extinction of conditioned fear. Physiol Rev. 90 (2), 419-463 (2010).

19. Sigurdsson, T. et al. Long-term potentiation in the amygdala: a cellular mechanism of fear learning and memory. Neuropharmacology. $\mathbf{5 2}$ (1), 215-227 (2007).

20. Bosch, D., Asede, D., Ehrlich, I. Ex-vivo optogenetic dissection of fear circuits in brain slices. J. Vis. Exp. (110) (2016).

21. Fenno, L., Yizhar, O., Deisseroth, K. The development and application of optogenetics. Annu Rev Neurosci. 34, 389-412 (2011).

22. Bienvenu, T.C.M., et al. Large intercalated neurons of amygdala relay noxious sensory information. J. Neurosci. 35 (5), $2044-2057$ (2015).

23. Sandri, C., Akert, K., Livingston, R.B., Moor, H. Particle aggregations at specialized sites in freeze-etched postsynaptic membranes. Brain Res. 41 (1), 1-16 (1972).

24. Likhtik, E., Popa, D., Apergis-Schoute, J., Fidacaro, G.A., Paré, D. Amygdala intercalated neurons are required for expression of fear extinction. Nature. 454 (7204), 642-645 (2008).

25. Fukazawa, Y., \& Shigemoto, R. Intra-synapse-type and inter-synapse-type relationships between synaptic size and AMPAR expression. Curr Opin Neurobiol. 22 (3), 446-452 (2012).

26. Amiry-Moghaddam, M., \& Ottersen, O.P. Immunogold cytochemistry in neuroscience. Nat Neurosci. 16 (7), $798-804$ (2013).

27. Fukazawa, Y., Masugi-Tokita, M., Tarusawa, E., Hagiwara, A., Shigemoto, R. SDS-digested Freeze-fracture replica labelling (SDS-FRL). In: Handbook of Cryo-Preparation Methods for Electron Microscopy. Cavalier A, et al., eds,, Boca Raton: CRC Press, 567-586 (2007).

28. Severs, N.J. Freeze-fracture electron microscopy. Nat Protoc. 2 (3), 547-76 (2007).

29. Tanaka, J. et al. Number and density of AMPA receptors in single synapses in immature cerebellum. J Neurosci. 25 (4), $799-807$ (2005). 
30. Mansouri, M. et al. Distinct subsynaptic localization of type 1 metabotropic glutamate receptors at glutamatergic and GABAergic synapses in the rodent cerebellar cortex. Eur J Neurosci. 41 (2), 157-167 (2015). 